\title{
Patterns of growth, oestradiol and progesterone released by in vitro cultured mouse ovarian follicles indicate consecutive selective events during follicle development
}

\author{
A. Fehrenbach ${ }^{1}$, N. Nüsse ${ }^{2}$ and P. L. Nayudu ${ }^{2 *}$ \\ ${ }^{1}$ Centre of Anatomy, Electron Microscopy, University of Göttingen, Kreuzbergring 36, D-37075 Göttingen, \\ Germany; and ${ }^{2}$ Department of Reproductive Biology, German Primate Centre, Kelinerweg 4, \\ D-37077 Göttingen, Germany
}

\begin{abstract}
This study investigated the relationship between individual follicle growth, steroid release and follicular morphology to provide basic information about critical stages in follicle development. Preantral mouse follicles secreted significant and constant amounts of progesterone that were not related to oestradiol production but did appear to be related to thecal organization. Oestradiol release was variable among follicles of equivalent diameter, but marked increases in oestradiol concentrations were measured in follicles of 300 and $400 \mu \mathrm{m}$ diameter. Over 4 days of culture, the proportion of follicles growing beyond the threshold diameter of $300 \mu \mathrm{m}$ was lower in small $(140-160 \mu \mathrm{m})$ than it was in large preantral follicles $(170-210 \mu \mathrm{m})$. Retardation of growth below this diameter was associated with significantly decreased steroid concentrations. In follicles growing beyond the threshold diameter, antrum formation progressed, but full Graafian stage was not attained. Among large follicles, variability in oestradiol release could not be associated with obvious histological differences. In contrast, structural disturbances were associated with reduced steroid production and growth abnormalities. Therefore, it is concluded that the preantral follicles selected for culture comprise distinct follicle populations with respect to their developmental potential in vitro, and that follicle development is controlled by endogenous processes involving oestradiol at distinct stages of follicle development.
\end{abstract}

\section{Introduction}

The in vitro culture of intact follicles has been used to investigate follicle dynamics under controlled conditions. Follicles are developmental units that, when isolated and taken into culture, represent an ideal model to study paracrine and autocrine regulation. In general, in vitro growth patterns of these follicles are similar to those observed in normal development in vivo (Pedersen, 1970; Nayudu and Osborn, 1992; Hartshorne et al., 1994a), although critical comparisons have not yet been performed. Meiotic competence of a proportion of the oocytes, induction of ovulation by $\mathrm{LH}$ and successful fertilization have all been demonstrated (Boland et al., 1993; Nayudu et al., 1994; Spears et al., 1994, 1996).

In all studies reported to date, release of oestradiol has been shown to increase progressively in the culture media of growing follicles and to be dependent on the presence and dose of FSH (Nayudu and Osborn, 1992; Cortvrindt et al., 1997) and cyclic AMP (Hartshorne et al., 1994b). Oestrogens have been acknowledged for many years to be intrafollicular organizers acting directly on granulosa cells during the preovulatory growth of the follicle (for review, see Boland and

*Correspondence

Received 6 October 1997
Gosden, 1994), but their paracrine and autocrine intrafollicular and intraovarian potential remain enigmatic. A tentative relationship has been established between secretion of oestradiol and follicle growth characteristics that are influenced by the diameter of the follicle selected for culture (Nayudu and Osborn, 1992). However, no critical studies have been performed that examine the basic parameters of follicular response in relation to critical transition phases of follicle development during which selection forces might be assumed to be at their most severe.

The quality of in vitro follicle development has been described with respect to antrum formation (Nayudu and Osborn, 1992) and oocyte maturation (Chouinard, 1975; van Blerkom and Runner, 1984; van Blerkom, 1989; Larsen et al., 1991; Hartshorne et al., 1994a). However, reliable grading criteria and critical characterization of what constitutes normal follicle development and dominant follicle quality, based on a combination of growth rate, steroid release and structural development of single follicles are required to enable critical evaluation of in vitro follicle culture.

Several modifications of in vitro culture of mouse ovarian follicles based on different criteria of follicle selection and culture conditions have been used by various workers (Nayudu and Osborn, 1992; Boland et al., 1993, 1994; Boland and Gosden, 1994; Hartshorne et al., 1994a, b; Almahbobi et al., 
1995). The differences in the techniques applied have led to some controversial results concerning patterns of growth and of steroid release in follicle development in vitro, and the variability of data has not been emphasized in most reports. Furthermore, some studies using follicle culture have reported low rates of ovulation (Boland et al., 1993; Nayudu, 1994) and a small proportion of follicles acquiring full meiotic competence in response to LH (Johnson et al., 1995), as well as low rates of embryo development (Spears et al., 1994), all of which suggest that follicle development in vitro is not yet optimal. Progesterone secretion was not detectable during the period of culture in follicles produced in vitro until recently (Hartshorne et al, 1994a). However, the increase in plasma progestin content during pro-oestrus and oestrus in mice and rats in vivo (Guttenberg, 1961; Butcher et al., 1974) suggests that progestins are secreted by the ovaries and are, therefore, an indicator of normal ovarian function.

A more critical analysis of the stage-specific relationships among variability in growth rates, oestradiol secretion patterns and degenerative events during follicle maturation is needed to provide a critical prerequisite for the correct interpretation of further studies. The aim of the present study was to identify and characterize critical steps of transition to improve the understanding of the physiological requirements for successful progression through these threshold developmental stages and, thereby, establish grading criteria on the basis of growth, steroid release and histology of in vitro cultured follicles. Further evaluation of the methods for assessing follicle development and steroid production was required to achieve this aim, with special focus being placed on the diversity within the follicle population selected for culture.

\section{Materials and Methods}

\section{Animals}

The 22 female prepubertal $\mathrm{C} 57 \mathrm{Bl} / 6 \mathrm{~J} \times \mathrm{CBA} / \mathrm{J}$ mice used in this study were bred in the German Primate Centre in Göttingen from parents obtained from Harlan Winkelmann, Borchen. The animals were provided with food and water ad libitum and housed in a temperature- and light-controlled room under a photoperiod of $12 \mathrm{~h}$ light: $12 \mathrm{~h}$ dark. Ovaries were excised from 21-23-day-old mice depending upon their body weight which ranged from 8.5 to $12 \mathrm{~g}$ on the day of the experiment. The experiments were performed in accordance with the German Animal Protection Laws.

\section{Isolation, preparation and in vitro culture of follicles}

The animals were anaesthetized with diethyl ether, and blood was obtained from the heart. The ovaries were removed aseptically and placed in Leibovitz L-15 medium (Gibco, Eggenstein) supplemented on the day of use with $1 \mathrm{mmol}$ glutamine $\mathrm{l}^{-1}$ (Gibco), $30 \mathrm{mg}$ BSA ml $\mathrm{m}^{-1}, 5 \mu \mathrm{g}$ human transferrin $\mathrm{ml}^{-1}$ (without iron), $2.5 \mu \mathrm{g}$ insulin $\mathrm{ml}^{-1}$ and $0.1 \mathrm{mg}$ vitamin $C \mathrm{ml}^{-1}$ (culture-grade chemicals, Sigma, St Louis, MO). Preparation, manual collection and selection of follicles were performed according to the method and criteria established by Nayudu and Osborn (1992) and Nayudu et al. (1994) but the culture was modified in the following way. Single follicles were cultured for 4 days (days $0-4$ ) in a humidified incubator at $37^{\circ} \mathrm{C}$ and gassed with $5.5-6.0 \% \mathrm{CO}_{2}$. Follicles with diameters between 140 and $210 \mu \mathrm{m}$ ( $\pm 5 \mu \mathrm{m}$ ) were selected for culture. The culture period of 4 days was chosen after preliminary studies had shown that the larger follicles ( $\geq 170 \mu \mathrm{m}$ at the start of the culture) had largely completed their development by this time, although the smaller starting diameter group required 5 days to reach an equivalent diameter. This choice of culture period ensured that the larger starting diameter follicles were not 'aged' at the end of culture and, therefore, could be compared for health with the smaller group.

A major change of method from that of previous studies was the use of hydrogel-coated, flat-bottomed 96-well microtitre plates (Costar, Bodenheim). The advantage of these plates is that the coating prevents cell attachment. The central eight wells (one row per day) were each filled with $40 \mu \mathrm{l}$ minimum essential medium ( $\alpha$ MEM; Gibco) supplemented with $2 \mathrm{mmol}$ glutamine $\mathrm{l}^{-1}, 10 \mu \mathrm{g}$ transferrin $\mathrm{ml}^{-1}, 5 \mu \mathrm{g}$ insulin $\mathrm{ml}^{-1}$, $0.1 \mathrm{mg}$ vitamin $\mathrm{C} \mathrm{ml}^{-1}$ (double the standard concentration required to reduce the number of follicles expelling oocytes during culture), $50 \mu \mathrm{l}$ mouse serum $\mathrm{ml}^{-1}$ and 1 iu human pituitary FSH $\mathrm{ml}^{-1}$ (Boehringer, Mannheim; manufacturer's information: immunoactivity $4500 \mathrm{iu} \mathrm{mg}^{-1} ; \mathrm{hTSH}<3 \%$; $\mathrm{hLH}<1 \%$ ). The medium powder was a special batch without vitamin $C$ and phenol red. Wells in one row that was not used for culture were each filled with $40 \mu \mathrm{l}$ phenol red containing media to monitor $\mathrm{pH}$ changes. Plates were not kept outside the incubator for longer than 2 min during handling to avoid $\mathrm{pH}$ changes. The base medium was prepared freshly every 2 weeks. The medium was not covered with oil, and the outer rows of wells contained sterile water to humidify the inner environment of the plate, preventing evaporation. This assured stability of medium osmolarity during a culture period of $24 \mathrm{~h}$ until transfer of follicles. Follicles were transferred with fine glass capillaries into the next row containing fresh medium, and the medium of the previous day was collected and stored individually at $-80^{\circ} \mathrm{C}$ until assayed for steroid hormones. Individual follicle growth patterns were obtained from daily measurement of follicle diameters (including theca layer) using a Zeiss stereomicroscope with ocular scales at a magnification of $\times 50$. Follicles observed to be about to extrude the oocyte by the movement of the oocyte to the periphery of the follicle were remeasured at shorter intervals until the oocyte was extruded so that the final diameter of the follicle could be determined.

\section{Fixation and processing of follicles}

The follicles were fixed overnight and postfixed for $2 \mathrm{~h}$ in individual microtitre plates using ascending concentrations of 1.0, 2.5 and $5.0 \%$ freshly prepared paraformaldehyde and $1 \%$ $\mathrm{OsO}_{4}$, respectively, all made up in 0.1 mol Hepes buffer $\mathrm{l}^{-1}$ at pH 7.4 (Merck, Darmstadt). Dehydration was performed through an ascending series of ethanol, immersion in propylene oxide and embedding in epoxy resin (Glycidether, Serva). An isector was used to generate isotropic uniform random sections according to the method of Nyengaard ef al. (1992). Semi-thin 
sections from nine representative follicles of different growth and hormone release patterns were cut using an ultracut (Jung RM2055; Leica, Hamburg) until the oocyte, germinal vesicle and nucleolus became visible and stained according to Richardson et al. (1960).

\section{Sample extraction and chromatography}

High pressure liquid chromatographic (HPLC) separations were performed on a pool of culture media to assess the specificity of the hormone measurements in the various assays used (see below) and to determine which oestrogens and progestins were being secreted by the follicles. Hormones were extracted by using diethyl ether and each extract was combined with 5000 c.p.m. of a mixture of $\left[{ }^{3} \mathrm{H}\right]$ steroids (oestrone, oestradiol and oestriol for HPLC of oestrogens; progesterone, 17 $\alpha$-hydroxyprogesterone, 20 $\alpha$-hydroxypregnenolone and pregnanediol for HPLC of progestins; Amersham Buchler, Braunschweig) as markers to indicate steroid elution positions. Steroids were separated by reverse-phase HPLC using a Nova-Pak C18 column $(3.9 \mathrm{~mm} \times 105 \mathrm{~mm}$; Millipore Corp., Milford, MA) and acetonitrile: water mixtures as eluents according to the method described by Heistermann et al. (1993).

\section{Hormone assays}

Progesterone, oestradiol and total oestrogen were determined directly from the culture media and from the HPLC elutions by enzyme immunoassays. The sensitivity of the progesterone assay was $0.12 \mathrm{ng} \mathrm{ml}^{-1}$ (hormone concentration at $90 \%$ binding) and all intra- and interassay coefficients of variance calculated from repeated measurements of quality controls were $<15 \%$. Immunoreactive oestradiol was measured by using an anti-17 $\beta$-oestradiol-6-CMO-BSA antibody (EO02; S. Klinger, St Albans) and oestradiol-6-glucuronide linked to alkaline phosphatase as label. The crossreactivities of the antiserum were $2 \%$ for oestrone, $1 \%$ for oestronesulfate, $<0.3 \%$ for all the other oestrogens and $<0.01 \%$ for all the other progestins tested. The sensitivity of the assay was $0.02 \mathrm{ng} \mathrm{ml}^{-1}$ and all intra- and interassay coefficients of variance were $<15 \%$. The total oestrogen immunoreactivity was measured by using an antibody raised in rabbits against oestradiol-17-hemisuccinate-BSA (gift from $H$. Meyer, Freising-Weihenstephan). The crossreactivities of the antiserum relative to oestradiol (100\%) were $110 \%$ for oestrone, $80 \%$ for $17 \alpha$-oestradiol, $2.5 \%$ for oestriol, $0.4 \%$ for oestradiol$17-\beta$-glucuronide and $<0.1 \%$ for all other steroids tested. The sensitivity of the assay was $0.08 \mathrm{ng} \mathrm{ml}^{-1}$ and all intra-assay coefficients of variation calculated from quality controls were $<10 \%$. In general, aliquots of $30 \mu \mathrm{l}$ of diluted samples (1:5; 1:10) were assayed in duplicate with the standards dissolved in media or assay buffer according to the sample tested after accuracy and linearity of the measurements in media had been confirmed. All assays were performed according to the method of Hodges et al. (1988).

\section{Statistical analysis}

Statistical analysis was performed by using SigmaStat for Windows 1.01. All values stated in the text are the mean $I S D$ of the grouped data and were compared by Kruskal-Wallis one way ANOVA on ranks followed by all pairwise multiple comparison procedures (Dunn's Method) or the MannWhitney rank sum test. A probability of $<0.05$ was accepted as significant. The comparison of dependent data, for example hormone release by the same follicle on consecutive days of the culture, were performed by repeated measures ANOVA on ranks. Correlation analysis was performed according to Spearman's rank correlation.

\section{Experimental design}

The major objective of this study was to describe the relationship between growth pattern and steroid release of follicles cultured under a standard set of non-restrictive conditions. The data were obtained and pooled from seven consecutive cultures and comprised a total of 152 follicles. Each ovary yielded about ten follicles acceptable for culture according to the criteria mentioned. A proportion of these follicles was used for the experiments presented here. Morphologically intact follicles were selected according to diameter and ranged from 140 to $210 \mu \mathrm{m}( \pm 5 \mu \mathrm{m})$. All follicles in the selected size range were in the preantral stage. The steroid profiles were based on the data of the 135 of these follicles for which all hormone values over the period of culture were available.

\section{Results}

\section{Variation in follicle growth pattern}

A classification system has been developed to provide a basis for the analysis of the observed variation in follicular growth. This system was grounded on initial and final follicle diameters as well as gross abnormalities, such as follicular degeneration or premature extrusion of the oocyte during the culture period. In 116 follicles that did not show either of these abnormalities, the initial diameter of each follicle was plotted individually against the final diameter (Fig. 1). The distribution pattern shows that final diameters ranged widely in follicles that were selected at diameters between 140 and $160 \mu \mathrm{m}$. In contrast, virtually all follicles starting at $\geq 170 \mu \mathrm{m}$ had a final diameter of $>300 \mu \mathrm{m}$ and showed a more restricted distribution of endpoint diameter than did the smaller follicles. Therefore, follicles were subdivided into two groups on the basis of starting diameters: $>160 \mu \mathrm{m}$ versus $\leq 160 \mu \mathrm{m}$. Follicles in the large preantral follicle group (LPF; $n=55$; $187 \pm 12 \mu \mathrm{m})$ reached a mean endpoint diameter of $358 \pm 33 \mu \mathrm{m}$. In the small preantral follicle group (SPF; $n=61$; $156 \pm 6 \mu \mathrm{m}$ ), the endpoint diameter of follicles $(309 \pm 42 \mu \mathrm{m}$ SD) was significantly lower.

The population was subdivided into two groups on the basis of the endpoint diameter exceeding or staying below $300 \mu \mathrm{m}$ to facilitate analysis of the variability within the group of smaller follicles (Fig. 2). Follicles that reached an endpoint diameter of $333 \pm 21 \mu \mathrm{m}$ were referred to as 'normal growth' small preantral follicles (ng-SPF: $n=39 ; 158 \pm 5 \mu \mathrm{m}$; Fig. $2 \mathrm{~b}$ ). Those follicles that reached significantly lower final diameters of $256 \pm 25 \mu \mathrm{m}$ were referred to as 'retarded growth' small preantral follicles (rg-SPF: $n=19 ; 151 \pm 7 \mu \mathrm{m}$; Fig. 2c). Follicles 


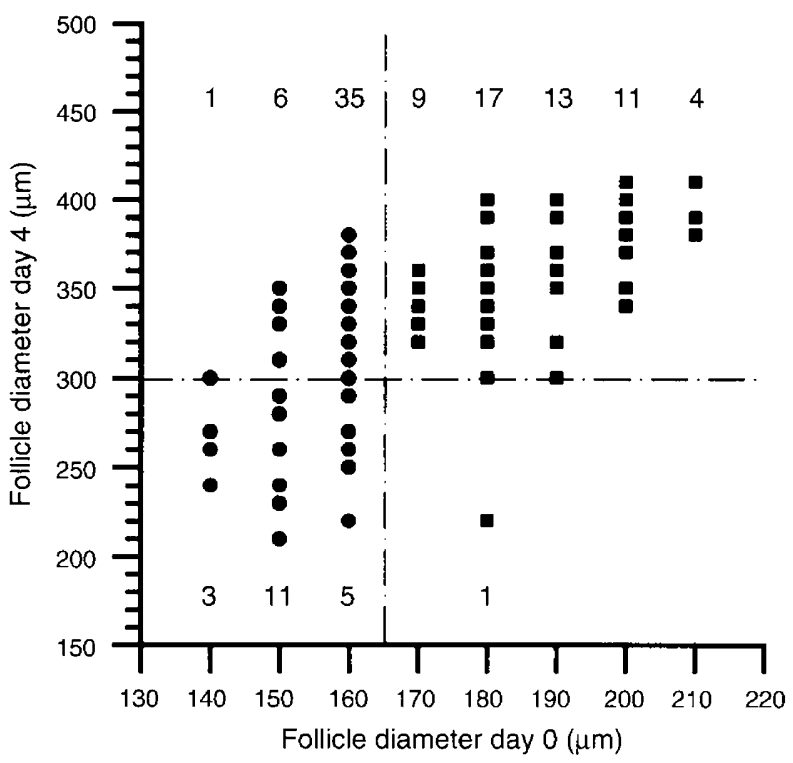

Fig. 1. Initial follicle diameter of 116 mouse follicles growing throughout a culture period of 4 days plotted against final follicle diameter. These data were used to divide follicles into small preantral follicles (SPF; $n=61$ ) and large preantral follicles (LPF; $n=55$ ) (vertical line). The numbers indicate the number of follicles that stayed below or exceeded a final diameter of $300 \mu \mathrm{m}$ (horizontal line).

from these two sub-groups did not differ in their initial diameters and retardation of growth became statistically significant on day 2 of the culture $(203 \pm 16 \mu \mathrm{m}$ in rg-SPF versus $235 \pm 20 \mu \mathrm{m}$ in $\mathrm{ng}$-SPF).

The pattern of follicle growth rates corroborates this division of the follicle population into the different groups. In rg-SPF, the growth rate decreased on day 2 and was reduced further to $27 \pm 15 \mu \mathrm{m}$ (Fig. 2f). However, in ng-SPF, the growth rates continued to increase and reached a maximum of $55 \pm 16 \mu \mathrm{m}$ on day 3 , and then decreased to $42 \pm 22 \mu \mathrm{m}$ on the last day of culture (Fig. 2e). A similar pattern of growth was observed in LPF (Fig. 2a), although growth rates of ng-SPF and LPF always differed significantly when the data from the same days of culture were compared. No significant difference was found between ng-SPF on day $2(46 \pm 20 \mu \mathrm{m})$ and LPF on day $I(44 \pm 19 \mu \mathrm{m})$ of the culture, indicating a $24 \mathrm{~h}$ shifted equivalence in growth characteristics that was maintained throughout the culture period.

Seven follicles (three SPF and four LPF) underwent degeneration, indicated by darkening of oocyte and granulosa cells. Regardless of starting diameter, degeneration was always accompanied by a retardation of or a halt in growth during culture. None of these follicles attained the threshold diameter of $300 \mu \mathrm{m}$. Oocyte extrusion was observed in 29 follicles, which were characterized further on the basis of the pattern of oestradiol release during culture (see description of oestradiol secretion patterns). In 17 of these follicles, oocyte extrusion (without the surrounding cumulus cells) was accompanied by undetectable oestradiol secretion (Fig. 3). In SPF, expulsion of oocytes occurred between day 2 and day 4 (Fig. 3b) and growth rates had decreased by day 2 of culture (Fig. 3e). In contrast, LPF grew normally up to day 2 and oocytes were expelled on days $2-3$ (Fig. 3a). These follicles did not grow beyond $300 \mu \mathrm{m}$ in diameter.

\section{Identification of secreted hormones}

High pressure liquid chromatographic separation profiles evaluated by immunoreactivity were determined from medium extracts (Fig. 4). Oestradiol and progesterone, identified by co-elution with tritiated authentic tracers, were confirmed as the only detectable form of oestrogen and progestin secreted by the follicles into the media. This finding was further supported by a comparative measurement of the total oestrogen and oestradiol in the non-extracted media of 20 follicles at day 4 . The total oestrogen concentrations were approximately $12 \%$ higher than those of oestradiol, which were within the range of the assay variability. In addition, the amounts of oestradiol and progesterone in undiluted mouse serum were determined from three serum pools collected from six, three and seven prepubertal females, respectively. Whereas oestradiol was below the detection limits of the assay, low but constant progesterone concentrations of $280 \mathrm{pg} \mathrm{ml}^{-1}$ were found in all serum samples tested. Therefore, the baseline steroid concentration in the mixture of culture medium was not measurable for oestradiol and was only $14 \mathrm{pg}$ per culture well ( $5 \%$ serum concentration) for progesterone.

\section{Pattern of in vitro progesterone release}

In normal and retarded growth follicles, progesterone production was stable for each individual follicle (Fig. $2 \mathrm{~g}, \mathrm{~h}, \mathrm{i}$ ). The highest concentrations of progesterone were released by LPF $\left(11.9 \pm 4.8 \mathrm{ng} \mathrm{ml}^{-1} ; n=41\right.$; Fig. $\left.2 \mathrm{~g}\right)$ which significantly exceeded the secretion rates by rg-SPF $\left(6.7 \pm 4.1 \mathrm{ng} \mathrm{ml}^{-1}\right.$; $n=19 ; \quad$ Fig. 2i). Progesterone production in ng-SPF $\left(9.5 \pm 4.3 \mathrm{ng} \mathrm{ml}^{-1} ; n=39\right.$; Fig. $\left.2 \mathrm{~h}\right)$ was not significantly different from either of the other groups. Follicles that degenerated $(n=7)$ showed variable progesterone release, decreasing to $<5 \mathrm{ng} \mathrm{ml}^{-1}$ as culture progressed. Inconsistent and low progesterone secretion was also found in the group of follicles $(n=17)$ in which extrusion of oocytes was accompanied by retarded development and lack of oestradiol in the culture media (Fig. 3). In contrast, follicles extruding oocytes but producing oestradiol in detectable amounts also released progesterone in low but stable concentrations of $5.5 \pm 3.1 \mathrm{ng}$ $\mathrm{ml}^{-1}(n=12)$ into the culture media (data not shown).

\section{Pattern of in vitro oestradiol release}

In contrast to secretion of progesterone, oestradiol secretion increased throughout in vitro culture (Fig. 2j,k,l). After the first $24 \mathrm{~h}$ of culture, oestradiol became detectable in the culture medium in approximately $7 \%$ of SPF and $50 \%$ of LPF. After $48 \mathrm{~h}$ of culture, detectable amounts of oestradiol were released by almost all LPF but only by $50 \%$ of ng-SPF and by $10 \%$ of rg-SPF. Whereas oestradiol secretion could be measured in all ng-SPF from the day 3 of culture, it remained undetectable in approximately $20 \%$ of rg-SPF. Although oestradiol release was variable among the follicles, hormone concentrations increased significantly every $24 \mathrm{~h}$ for the whole period of culture in each 

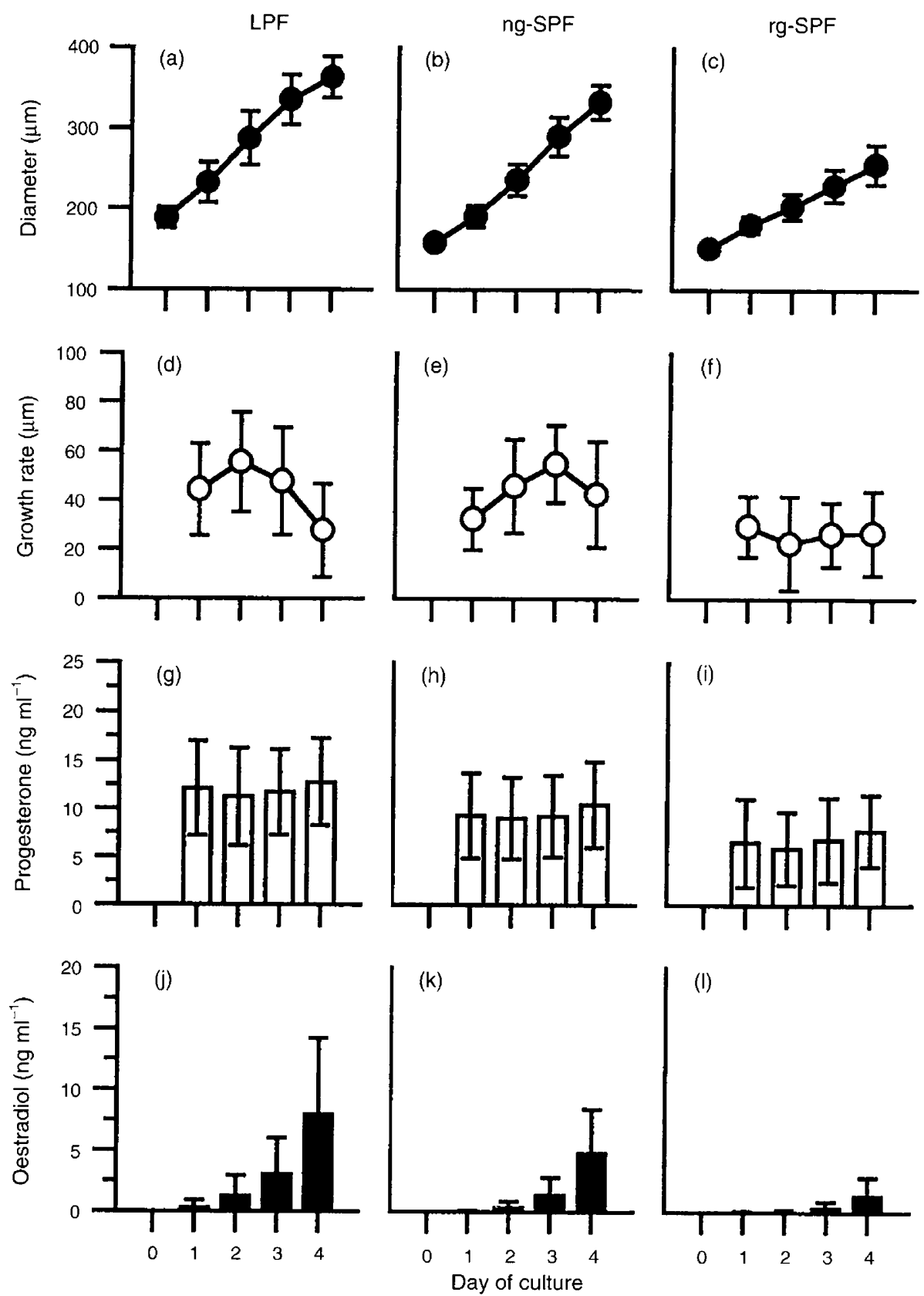

Fig. 2. Mean $\pm \mathrm{SD}(\mathrm{a}, \mathrm{b}, \mathrm{c})$ diameter, $(\mathrm{d}, \mathrm{e}, \mathrm{f})$ growth rate, $(\mathrm{g}, \mathrm{h}, \mathrm{i})$ progesterone and $(\mathrm{j}, \mathrm{k}, \mathrm{l})$ oestradiol release of 99 in vitro cultured mouse follicles for which all parameters were available for all 4 days of culture. $(\mathrm{a}, \mathrm{d}, \mathrm{g}, \mathrm{j})$ Large preantral follicles (LPF; $n=4 \mathrm{I}$ ) (b,e,h,k), normal growth small preantral follicles (ng-SPF $n=39$ ) and $(c, f, i, l)$ retarded growth small preantral follicles (rg-SPF; $n=19$ ) were categorized according to their growth rates and final diameters remaining below or exceeding $300 \mu \mathrm{m}$ on day 4 of culture. Progesterone and oestradiol concentrations were measured in culture media collected each day.

of the follicle populations. Oestradiol secretion of LPF significantly exceeded that of SPF on the same days of culture and, by day 4 of culture, reached $7.9 \pm 6.2 \mathrm{ng} \mathrm{ml}^{-1}$ (Fig. 2j), $4.8 \pm 3.6 \mathrm{ng} \mathrm{ml}^{-1}$ (Fig. 2k) and $1.3 \pm 1.5 \mathrm{ng} \mathrm{ml}^{-1}$ (Fig. 2l) in LPF, ng-SPF and $\mathrm{rg}$-SPF, respectively. The apparent $24 \mathrm{~h}$ shift in follicle growth between ng-SPF and LPF is paralleled in the pattern of secretion of oestradiol, with similar amounts of oestradiol in the media of ng-SPF to those measured in LPF on the previous day.
Oestradiol concentrations remained low or undetectable in follicles that degenerated during in vitro culture. The same phenomenon was found in $60 \%$ of follicles that grew and subsequently extruded their oocytes (Fig. 3). On the basis of oestradiol secretion patterns, these follicles were subdivided into two groups. One group comprised follicles $(n=17)$ with more or less undetectable oestradiol (Fig. 3g,h). The follicles of the other group $(n=12)$ all showed measurable hormone secretion patterns similar to those observed in ng-SPF and LPF 

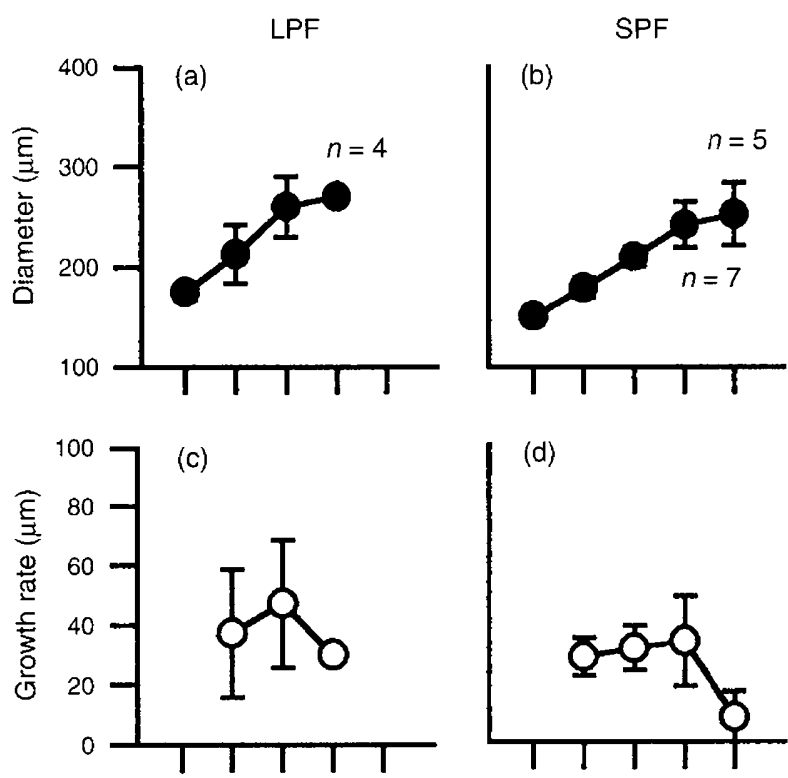

(d)

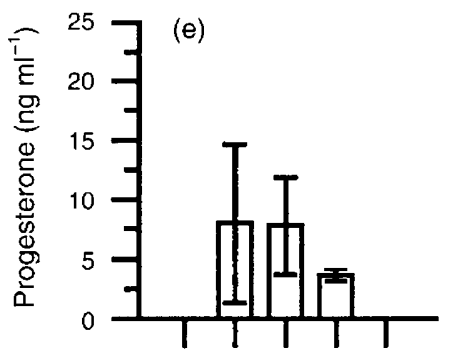

(f)

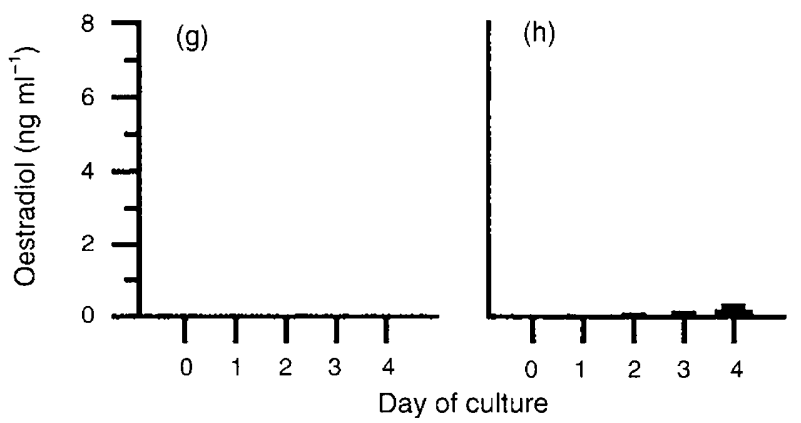

Fig. 3. Mean $\pm \mathrm{SD}(\mathrm{a}, \mathrm{b})$ diameter, $(\mathrm{c}, \mathrm{d})$ growth rate, $(\mathrm{e}, \mathrm{f})$ progesterone and ( $\mathrm{g}, \mathrm{h}$ ) oestradiol release of in vitro cultured large (LPF; $n=7 ; \mathrm{a}, \mathrm{c}, \mathrm{e}, \mathrm{g}$ ) and small (SPF; $n=10 ; \mathrm{b}, \mathrm{d}, \mathrm{f}, \mathrm{h}$ ) preantral mouse follicles that prematurely extruded the oocytes. Numbers indicate those follicles that remained intact to this day of culture.

(Fig. 2), indicating that, in this group, oocyte extrusion could have been due to damage during handling.

\section{Growth-hormone relationships}

Follicle diameter and oestradiol release both showed a significant increase each day. The final diameters of 99 follicles (small and large preantrals) were correlated with the corresponding amounts of oestradiol secreted on day 4 (Fig. 5). The correlation was performed separately for LPF, ng-SPF and rg-SPF. No significant relationship was found between follicle
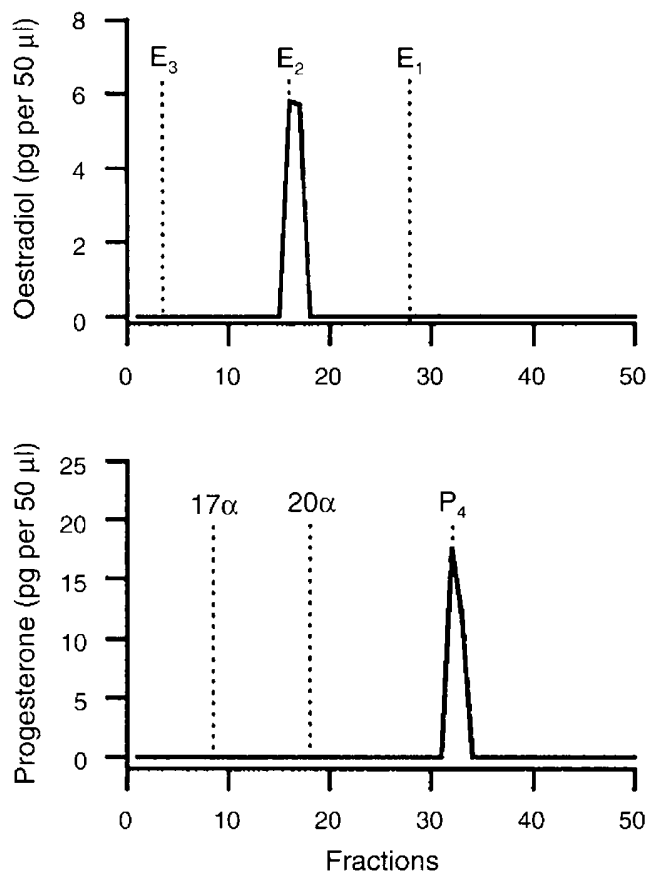

Fig. 4. High pressure liquid chromatographic separation of oestrogens and progestins obtained from medium extract in which mouse follicles had been cultured for 4 days. Immunoreactivity measured in each fraction is compared with peak recovery of radioactive markers indicated by letters. $\mathrm{E}_{1}$ : oestrone; $\mathrm{E}_{2}$ : oestradiol; $\mathrm{E}_{3}$ : oestriol; $17 \alpha$ : $17 \alpha$-hydroxyprogesterone; $20 \alpha$ : $20 \alpha$-hydroxypregnenolone; $\mathrm{P}_{4}$ : progesterone. The presence of one immunoreactive peak in each assay indicated the high specificity for the measurements of oestradiol and progesterone.

diameter and oestradiol release in $\operatorname{rg}-\mathrm{SPF}(n=21)$ but, in ng-SPF $(n=39)$ and LPF $(n=41)$, correlation was significant at $P=0.01$ and $P=0.001$, respectively. This is due, at least in part, to the fact that in the LPF group, five of the ten largest follicles $(>380 \mu \mathrm{m})$ secreted very high concentrations of oestradiol (>10 $\mathrm{ng} \mathrm{ml}^{-1}$ ) on day 4 . In all other LPF, ranges of diameter $(300-380 \mu \mathrm{m})$ and oestradiol concentrations $\left(0.5-10.0 \mathrm{ng} \mathrm{ml}^{-1}\right)$ overlapped with those in ng-SPF.

\section{Structural integrity of follicles after in vitro culture}

Histological examination was performed on a random sample of six normal growing follicles in the diameter range $360-410 \mu \mathrm{m}$ to examine whether differences in oestradiol concentrations were related to any morphological feature. Although there were major differences in oestradiol concentrations (range $3.2-27.0 \mathrm{ng} \mathrm{ml}^{-1}$ ), associated systematic histological differences could not be identified. This was probably due to the simultaneous appearance of structural characteristics indicating both progressed and retarded development in the individual follicles. An advanced state of follicular development achieved during 4 days of culture and originating from a follicle $\geq 170 \mu \mathrm{m}$ at the start of culture is shown (Fig. 6a,c,e). Advanced development is indicated by an oocyte diameter $>70 \mu \mathrm{m}$, an invaginated germinal vesicle, progressed antrum formation, 1-3 layers of cuboidal cumulus cells intimately 


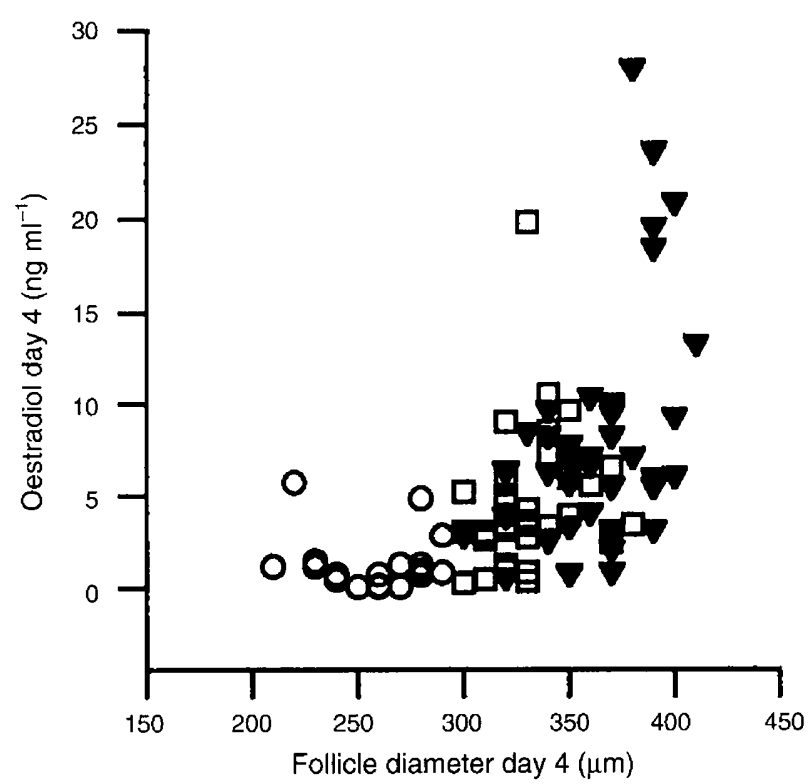

Fig. 5. Relationship between follicle diameters and oestradiol concentrations measured on day 4 of in vitro culture of mouse follicles. Pearson correlation was performed separately for the three different follicle groups $(\boldsymbol{\nabla}$, large preantral follicles (LPF), $n=41 ; 0$, retarded growth small preantral follicles (rg-SPF), $n=19 ; \square$, normal growth small preantral follicles (ng-SPF), $n=39$ ). The relationship was not significant between the pair of variables in either the rg-SPF or the ng SPF group $(\Gamma>0.05)$ whereas a correlation cocfficient of 0.5 was calculated for the LPF group $(P<0.05)$.

surrounding the oocyte and sending cytoplasmic projections into a homogeneously stained zona pellucida (Fig. 6c). In contrast, in the same follicle, incomplete maturation is indicated by an apparently unrimmed nucleolus and clumped distribution of the dark ooplasmic granules. In five of the six follicles examined, full Graafian stage had not been attained and the cumulus-oocyte complex was still held in the centre of the antral cavity by cords of granulosa cell mass (Fig. 6a).

The characteristics of follicular disintegration, such as apoptotic cells or nucleolar pycnosis, were seen only in one follicle (Fig. 7a,c,e,f). This was a large follicle (starting diameter $\geq 170 \mu \mathrm{m}$ ) that had attained the Graafian stage (with the oocyte no longer positioned centrally and an undivided antral cavity). In this follicle, the oocyte diameter was approximately $60 \mu \mathrm{m}$, and ooplasmic granules were dispersed and unclustered among prominent vacuoles. The chromatin within the germinal vesicle was highly but irregularly condensed, there were no cytoplasmic projections from cumulus cells into the zona pellucida, and a large proportion of apoptotic cells among the cumulus complex was indicated by progressed condensation of chromatin into crescentic caps at the periphery of the nucleus (Fig. 7a,c). This follicle grew continuously throughout the culture period from 180 to $400 \mu \mathrm{m}$ and secreted relatively high concentrations of progesterone $\left(15.2 \mathrm{ng} \mathrm{ml}^{-1}\right.$ ) on day 4 , whereas oestradiol concentrations of approximately $6.1 \mathrm{ng}$ $\mathrm{ml}^{-1}$ were within the lower range of amounts secreted by this size class. The appearance of this follicle suggests that it had already completed its development before day 4 of culture and had commenced the early stages of degeneration due to ageing.
However, retarded growth $(n=1$; Fig. $6 \mathrm{~b}, \mathrm{~d}, \mathrm{f})$ and initiation of oocyte extrusion ( $n=2$; Fig. $7 \mathrm{~b}, \mathrm{~d}$ ) were accompanied by barely detectable oestradiol secretion $\left(0.2-0.6 \mathrm{ng} \mathrm{ml}^{-1}\right)$ which was associated with structural disintegration. In the three representative follicles sectioned, antral formation was restricted to some small cavities among the central zone of the granulosa layer (Figs $6 \mathrm{~b}$ and $7 \mathrm{~b}$ ). The highly condensed nucleolar mass (rimmed stage) was surrounded by an irregularly shaped halo of moderately condensed chromatin material (Figs $6 \mathrm{~d}$ and $7 \mathrm{~d}$ ), indicating incomplete nuclear maturation. Differences between the retarded growth follicle and the two follicles that were in the process of extruding their oocytes mainly concerned the appearance of the cumulus cells, granulosa cells and ooplasma. In the follicles that were extruding their oocytes, the cumulus and granulosa cells appeared swollen and the ooplasma was vacuolated and lacked dark granules, giving an appearance that suggested oocyte degeneration (Fig. 7d). These structures did not appear to be affected in the oocyte of the retarded growth follicle, which showed a dispersed distribution of dark granules (Fig. 6d,f), although cumulus cells displayed apoptotic chromatin disturbances and intercellular spaces were widened among the granulosa masses and the cells of the theca interna (Fig. $6 \mathrm{~d}, \mathrm{f}$ ).

In general, the thecal cells appeared normally differentiated for all follicles sectioned (Fig. 6b). High progesterone concentrations ( $>17 \mathrm{ng} \mathrm{ml}^{-1}$ ) were measured in five follicles in which parts of the theca were extremely proliferated and reached a thickness of up to $00 \mu \mathrm{m}$ (Fig. 7e). 'I hecal cells were irregularly shaped and vacuolated, in loose contact with each other and with the adjoining theca interna, and contained large aggregations of lipids identified by their yellow shimmering appearance. In some small areas, the theca was limited by a border of epithelial-like cells (Fig. $7 \mathrm{f}$ ) but, in general, its outer borders appeared rather irregular.

\section{Discussion}

This study has characterized variation in in vitro follicle development by analysis of the relationships among the parameters of follicle growth, histology and steroid release into the culture media. It has been shown for the first time that, in mouse ovarian follicles, the major secreted forms of oestrogens and progestins are oestradiol and progesterone.

Oestradiol is considered to be an important parameter indicating progressive maturation during early-to-mid in vitro follicle development. On the basis of in vitro follicle growth rates and oestradiol secretion patterns, SPFs appear to develop into LPFs within $24 \mathrm{~h}$. In the present study, SPFs corresponded to type $4 / 5$ a follicles and LPFs to type $5 b$ follicles according to the classification system of Pedersen and Peters (1968) and Pederson (1970) which divides follicles into eight stages. Those follicles that were started in culture at stage $4 / 5$ a displayed a greater range in final diameter than did the $5 b$ follicles, which tended to grow more uniformly (Nayudu and Osborn, 1992). Therefore, although classified into the same stage by Pederson and Peters (1968), 5a and 5b follicles may represent two distinct populations that differ in the development of some stage-specific unknown characteristic supporting continuous growth into the antral stages. Selective events during 

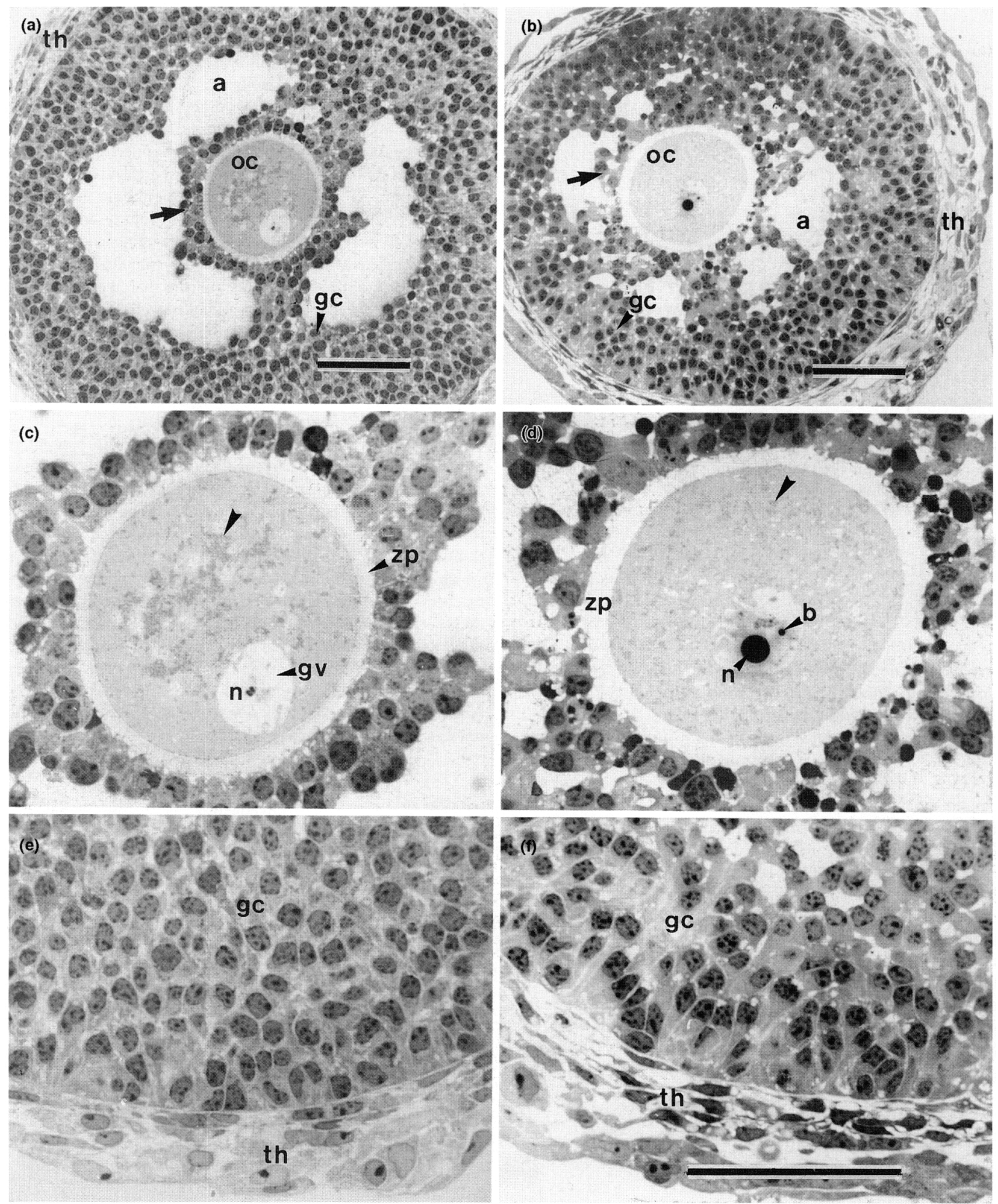

Fig. 6. Semi-thin sections $(1 \mu \mathrm{m})$ of mouse ovarian follicles grown from the large preantral stage $(>170 \mu \mathrm{m})$ and fixed after 4 days in culture. $(a, c, e)$ Follicle with a final diameter of about $370 \mu \mathrm{m}$, and $(\mathrm{b}, \mathrm{d}, \mathrm{f})$ follicle with a final diameter not exceeding $300 \mu \mathrm{m}$ in diameter. Antral cavities (a) and oocyte (oc) in a central position surrounded by the zona pellucida (zp) and cumulus cells (arrows); nucleolus (n) in the germinal vesicle (gv); dark ooplasmic granules corresponding to mitochondria (arrowheads); granulosa cells (gc); theca (th) consisting of one or two inner cell layers and an outer thicker sheath of cells; extranucleolar body (b). Scale bars represent $50 \mu \mathrm{m}$, with the same magnification in $\mathrm{c}-\mathrm{f}$. 


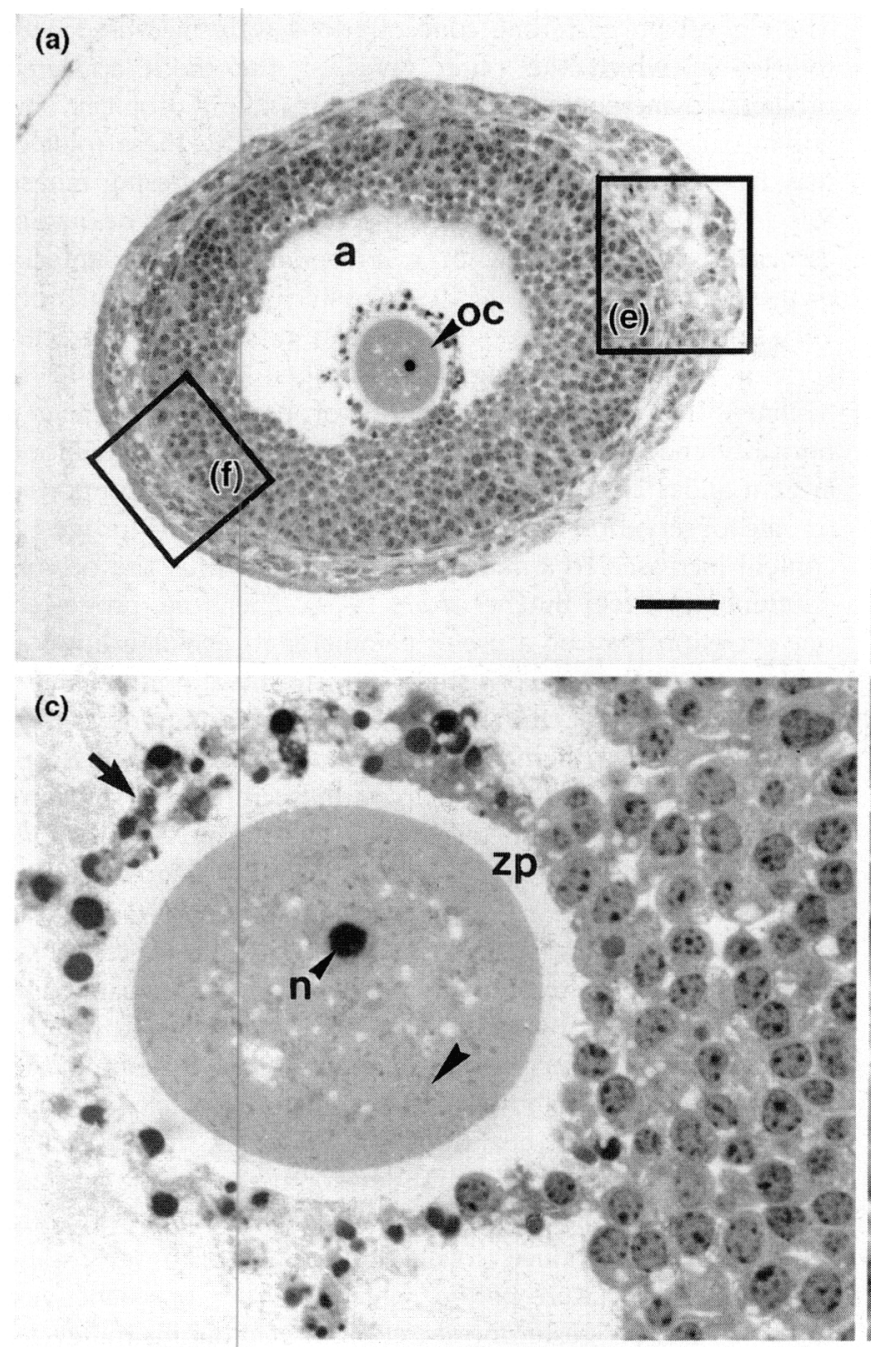

(b)
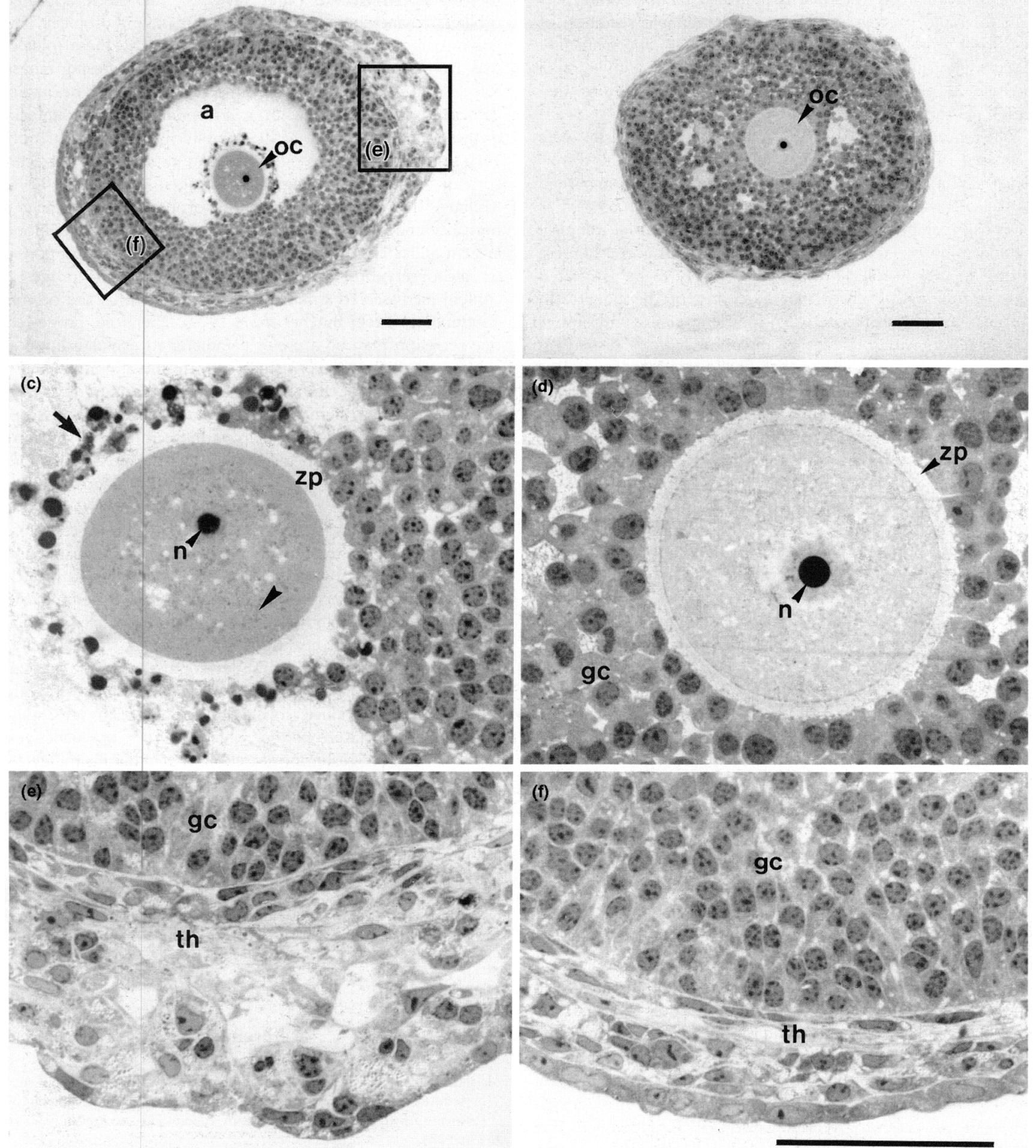

Fig. 7. Semi-thin sections ( $1 \mu \mathrm{m}$ ) of mouse ovarian follicles fixed after 4 days in culture. $(a, c, e, f)$ Apoptotic Graafian follicle grown from the large preantral stage to $400 \mu \mathrm{m}$ in diameter, and $(b, d)$ follicle grown from the small preantral stage to $280 \mu \mathrm{m}$ that prematurely started to extrude the oocyte on day 4. a, Antrum; gc, granulosa cells; $n$, nucleolus; oc, oocyte; th, theca; zp, zona pellucida. Arrowheads indicate dark ooplasmic granules and arrow indicates an apoptotic cumulus cell. Scale bars represent $50 \mu \mathrm{m}$, with the same magnification in c-f. 
early-to-mid-stages in follicle maturation appear to occur at the transition from type $4 / 5$ a to $5 b$ follicles, with a threshold diameter of approximately $180 \mu \mathrm{m}$. Successful transition through this earlier threshold is indicated by the commencement of rapid growth, the onset of measurable secretion of oestradiol and with antrum formation at approximately $300 \mu \mathrm{m}$. Antrum formation itself is associated with a rapid increase in oestradiol secretion on day 3 of culture that represents the transition to type 6 follicles.

When normal and abnormal growth pattern of follicles were compared, a clear relationship between histological disturbances and a decrease in oestradiol production was established for follicles with retarded and degenerative growth as well as for those that prematurely extruded the oocyte. Histological differences observed for typical examples of this group suggest distinct failures in follicle development likely to be associated with retardation of growth or extrusion of the oocyte. In contrast, no relationship between follicle structure and oestradiol secretion was found in examples of follicles displaying normal growth, and follicles that finally produced excessive amounts of oestradiol did not appear to be histologically different from those secreting lower oestradiol concentrations. Irrespective of the amount of oestradiol secretion, individual follicles showed a mosaic of histological features and there was a degree of asynchrony in the various compartments and functions of the oocyte and follicle. For example, characteristics of a progressed state of maturation, like mitochondrial clustering (van Blerkom and Runner, 1984), could appear in combination with a decondensed (unrimmed) nucleolus indicating a retarded state (Chouinard, 1975). However, a 2-5-fold increase in oestradiol production between day 3 and day 4 observed in some of the largest follicles attaining $400 \mu \mathrm{m}$ in diameter, appeared to be indicative of final maturation changes. This suggests that oestradiol is an indicator of dominant follicle development, the morphological indications of which become evident only later.

It has been shown for the first time that progesterone was secreted by isolated mouse ovarian follicles in significant and constant amounts throughout the culture period. Smyth $e t$ al. (1994) found that the pattern of progesterone released by in vitro cultured rat follicles was not as prominent and constant as that of mouse follicles in the present study, possibly as a result of the different culture conditions used. Among follicles categorized as growing normally in culture in the present study, progesterone secretion was not correlated with oestradiol production, growth rate or state of maturation, indicating a production process that may be independent of granulosa cell function. In consequence, progesterone secretion could not be associated with luteinization and must be considered as a normal attribute of mouse follicles. This suggestion is supported by a report that in vitro superfused slices of rat ovarian tissue released detectable amounts of progestins at all stages of the oestrous cycle (Anderson et al., 1973). However, the reported increase in progestin secretion and associated decrease in oestrogen release by clumps of follicles towards the end of a much longer culture period of mouse ovarian follicles (Qvist et al., 1990) is strongly suggestive of luteinization.

In contrast, abnormal follicle development in the present study was associated with both low progesterone values and reduced oestradiol production, as well as disturbances in thecal histology indicating that normal granulosa cell function is associated with basement membrane and theca cell integrity. The highest progesterone concentrations were measured with follicles in which the outer layer of the theca appeared proliferated and contained large amounts of lipid droplets. This finding suggests that progesterone released by these follicles may have been of thecal origin. Previous studies using mouse follicle culture have reported high concentrations of oestradiol associated with very low or even undetectable amounts of progesterone (Boland et al., 1993) which suggests that only very small amounts of progesterone are necessary as a precursor for androgen leading to oestrogen production. It is assumed that the higher amounts of progesterone have a primarily endocrine function and were secreted into the blood system under in vivo conditions. This assumption is supported by the observation that, in mice and rats, plasma progestin content increased to a maximum during proestrus and oestrus (Guttenberg, 1961; Butcher et al., 1974). Therefore, progesterone secretion may be a useful parameter to evaluate how far culture conditions are consistent with the situation in vivo.

On the basis of the results of the present study, it is hypothesized that there are critical phases in follicle development over threshold steps. Two of these threshold steps are proposed to be between the small and large preantral stages defined above, and between the large preantral stage the phase of antral development. Furthermore, passing between these different stages may be associated with increased selective pressure, indicated by the greater developmental variability among the group of smaller follicles. It is suggested that the timing of the initial secretion of oestradiol may be an important indicator for a normally developing preantral follicle, and that production of large quantities of oestradiol may support normal transition through antral development into the preovulatory stage. It is also reported for the first time that growing mouse follicles produce constant amounts of progesterone throughout the culture period, which may be consistent with the pattern of progesterone secretion in vivo during the cycle.

The authors wish to thank P. Kiesel for her assistance with the follicle culture, A. Wienecke for her assistance in HPLC, M. Heistermann for providing us with the assays for hormone measurements, J. Giebel for kindly supporting the histological studies and M. Gore for valuable discussions. This study was supported by Bundesinstitut für gesundheitlichen Verbraucherschutz und Verterinärmedizin (BgVV), post box 330013, D-14191 Berlin, Germany.

\section{References}

Almahbobi G, Nagodavithane A and Trounson AO (1995) Effects of epidermal growth factor, transforming growth factor $\alpha$ and androstendione on follicular growth and aromatization in culture Human Reproduction 10 2767-2772

Anderson LM, Turnipseed MR and Ungar F (1973) Progestin production during superfusion of ovaries from cycling rats Endocrinology $92 \quad 265-274$

Boland NI and Gosden RG (1994) Effects of epidermal growth factor on the growth and differentiation of cultured mouse ovarian follicles Journal of Reproduction and Fertility 101 369-374

Boland NI, Humpherson PG, Leese HJ and Gosden RG (1993) Pattern of lactate production and steroidogenesis during growth and maturation of mouse ovarian follicles in vitro. Biology of Reproduction 48 798-608 
Boland NI, Humpherson PG, Leese HJ and Gosden RJ (1994) The effect of glucose metabolism on murine follicle development and steroidogenesis in vitro. Human Reproduction 9 617-623

Butcher RL, Collins WE and Fugo NW (1974) Plasma concentration of LH, FSH, prolactin, progesterone and oestradiol- $17 \beta$ throughout the 4-day estrous cycle of the rat Endocrinology 94 1704-1708

Chouinard LA (1975) A light- and electron-microscope study of the oocyte nucleus during development of the antral follicle in the prepubertal mouse Journal of Cell Science 17 589-615

Cortvrindt R, Smitz J and Van Steirteghem AC (1997) Assessment of the need for follicle stimulating hormone in early preantral mouse follicle culture in vitro. Human Reproduction 12 759-768

Guttenberg I (1961) Plasma levels of 'free' progestin during the estrous cycle in the mouse Endocrinology 68 1006-1009

Hartshorne GM, Sargent IL and Barlow DH (1994a) Meiotic progression of mouse oocytes throughout follicle growth and ovulation in vitro. Human Reproduction 9 352-359

Hartshorne GM, Sargent IL and Barlow DH (1994b) Growth rates and antrum formation of mouse ovarian follicles in vitro in response to folliclestimulating hormone, relaxin, cyclic AMP and hypoxanthine Human Reproduction 9 1003-1012

Heistermann M, Tari S and Hodges JK (1993) Measurement of fecal steroids for monitoring ovarian function in New World primates. Callitrichidae Journal of Reproduction and Fertility $99243-251$

Hodges JK, Green DI, Cottingham PG, Sauer MJ, Edwards C and Lightman SL (1988) Induction of luteal regression in the marmoset monkey (Callithrix jacchus) by a gonadotropin releasing hormone antagonist and the effects on subsequent follicular development Journal of Reproduction and Fertility $\mathbf{8 2}$ 743-752

Johnson LD, Albertini DF, McGinnis LK and Biggers JD (1995) Chromatin organization, meiotic status and meiotic competence acquisition in mouse oocytes from cultured ovarian follicles Journal of Reproduction and Fertility $104 \quad 277-284$

Larsen WJ, Wert SE, Chen L, Russell P and Hendrix EM (1991) Expansion of the cumulus-oocyte complex during the preovulatory period: possible roles in oocyte maturation, ovulation, and fertilization. In Ultrastructure of the Ovary pp 45-61 Eds G Familiari, S Makabe and PM Motta. Kluwer, Dordrecht
Nayudu PL (1994) In vitro growth of oocytes. The use of immature human oocytes for reproductive toxicology: potential risks and benefits Human Reproduction 9 1585-1588

Nayudu PL and Osborn SM (1992) Factors influencing the rate of preantral and antral growth of mouse ovarian follicles in vitro. Journal of Reproduction and Fertility 95 349-362

Nayudu PL, Kiesel PS, Nowshari MA and Hodges JK (1994) Abnormal in vitro development of ovarian follicles explanted from mice exposed to tetrachlorvinphos Reproduction and Toxicology 8 261-268

Nyengaard J, Jorgen H and Gundersen G (1992) The isector: a simple and direct method for generating isotropic, uniform random sections from small specimens Journal of Microscopy 165 427-431

Pedersen T (1970) Follicle kinetics in the ovary of the cyclic mouse Acta Endocrinologica $64 \quad 304-323$

Pedersen T and Peters H (1968) Proposal for a classification of oocytes and follicles in the mouse ovary Journal of Reproduction and Fertility 17 555-557

Qvist R, Blackwell LF, Bourne H and Brown JB (1990) Development of mouse ovarian follicles from primary to preovulatory stages in vitro. Journal of Reproduction and Ferfility 89 169-180

Richardson KC, Jarett L and Finke EH (1960) Embedding in epoxy resins for ultrathin sectioning in electron microscopy Stain Technology 35 313-323

Smyth CD, Gosden RG, McNeilly AS and Hillier SG (1994) Effect of inhibin immunoneutralization on steroidogenesis in rat ovarian follicles in vitro. Journal of Endocrinology 140 437-443

Spears N, Boland NI, Murray AA and Gosden RG (1994) Mouse oocytes derived from in vitro grown primary ovarian follicles are fertile Human Reproduction 9 527-532

Spears N, de Bruin JP and Gosden RG (1996) The establishment of follicular dominance in co-cultured mouse ovarian follicles Journal of Reproduction and Fertility 106 1-6

van Blerkom J (1989) Morphodynamics of nuclear and cytoplasmic reorganization during the resumption of arrested meiosis in the mouse oocyte. In Development of Preimplantation Embryos and their Environment pp 33-51 Eds $\mathrm{K}$ Yoshinga and T Mori. Alan R. Liss, New York

van Blerkom J and Runner MN (1984) Mitochondrial reorganization during resumption of arrested meiosis in the mouse oocyte American Journal of Anatomy 171 335-355 